Nota de investigación

\title{
Variabilidad genotípica de aislados de Phytophthora capsici en Guanajuato
}

\author{
Brenda Zulema Guerrero-Aguilar ${ }^{1}$ \\ José Luis Pons-Hernández ${ }^{1}$ \\ Mario Martin González-Chavira ${ }^{1 \S}$ \\ Enrique González-Pérez ${ }^{1}$ \\ Salvador Villalobos-Reyes ${ }^{1}$ \\ Raúl Rodríguez Guerra ${ }^{2}$
}

${ }^{1}$ Centro de Investigación Regional del Centro-INIFAP. Carretera Celaya-San Miguel de Allende km 6.5, Celaya, Guanajuato, México. CP. 38124. (guerrero.brenda@inifap.gob.mx; pons.joseluis@inifap.gob.mx; gonzalez.enrique@inifap.gob.mx; villalobos.salvador@inifap.gob.mx ). ${ }^{2}$ Centro de Investigación Regional del Noreste-INIFAP. Carretera Matamoros Reynosa km 61, Col. Zona Rural Rio Bravo, Rio Bravo, Tamaulipas, México. CP. 88900. (rodriguez.raul@inifap.gob.mx).

${ }^{\S}$ Autor para correspondencia: gonzalez.mario@inifap.gob.mx.

\section{Resumen}

Phytophthora capsici, es el agente causal de la 'marchitez del chile' una de las enfermedades más importantes de este cultivo. Para establecer estrategias de control eficientes es necesario el conocimiento de la diversidad del patógeno, para esto, se realizó esta investigación cuyo objetivo fue determinar el grado de diversidad genética de 30 aislados monozoospóricos obtenidos de chile (Capsicum annuum L.) de seis municipios del estado de Guanajuato, y dos cepas de referencia. La variación genética se determinó mediante la técnica de AFLP (en español: polimorfismo en la longitud de fragmentos amplificados). La similitud encontrada entre los aislados va de 76 a $95 \%$. Los resultados obtenidos indican que dentro de la población se forman grupo basados en las localidades de aislamiento. No se encontraron organismos clónales por lo que los 32 aislamientos analizados fueron genotipos únicos, y no se encontró ninguna correlación entre grupos definidos por marcadores moleculares y virulencia, tipo de apareamiento o respuesta a metalaxil.

Palabras clave: diversidad poblacional, marchitez del chile, variación genética.

Recibido: enero de 2022

Aceptado: febrero de 2022 
El oomiceto Phytophthora capsici Leonian, es un patógeno hemibiotrófico que causa epidemias graves en una amplia gama de cultivos en todo el mundo (Lamour et al., 2012). En el cultivo de chile (Capsicum annuum L.), forma parte del complejo causante de la secadera o marchitez del chile (Erwin y Ribeiro, 1996), que en México y específicamente en el estado de Guanajuato, es una de las principales causas de pérdida del cultivo (González-Pérez et al., 2004), llegando a ser ésta hasta de $100 \%$ cuando las condiciones ambientales son favorables para el desarrollo de este patógeno (Universidad Illinois, 2001).

En el campo, el manejo de esta enfermedad se basa principalmente en la aplicación de fungicidas, en combinación con prácticas culturales que limitan el desarrollo de la marchitez, como camas elevadas, acolchado plástico y riego por goteo entre otras (Granke et al., 2012). Sin embargo, la amplia gama de huéspedes de $P$. capsici, su capacidad de recombinación sexual y la producción de oosporas como estructuras de supervivencia, ha limitado la eficacia de las estrategias de control (McDonald y Linde, 2002; Gobena et al., 2012). Particularmente, la recombinación sexual entre los tipos de apareamiento A1 y A2 de este oomiceto heterotálico, puede generar nuevos genotipos con mayor virulencia, patogenicidad, resistencia a fungicidas y capacidad para superar la resistencia del huésped (Granke et al., 2012).

Los esporangios de $P$. capsici se dispersan con poca frecuencia entre los campos por el viento (Lamour y Hausbeck, 2001b). En cambio, se sabe que el movimiento de fuentes de agua superficial para riego y el movimiento de material vegetal infectado o suelo infestado, son factores clave en la propagación local de P. capsici (Granke et al., 2009). Estas condiciones limitan la propagación rápida a largas distancias de este patógeno, por lo que se esperaría que un mejor conocimiento de la diversidad genética regional de la población de patógenos permitiría un despliegue más efectivo de variedades resistentes y el establecimiento de programas de mejoramiento que busquen generar una resistencia duradera del huésped (Quesada-Ocampo et al., 2011).

Esta evaluación de la diversidad genética se basa en diferentes características, entre ellas las moleculares (Martin et al., 2012). Las características moleculares son datos basados en ácido desoxirribonucleico (ADN) que se han usado para determinar la diversidad genética (Mohammadi y Prasanna, 2003), entre otras razones por que esta información no está influenciada por condiciones ambientales. Para generar estos datos, existen diferentes protocolos siendo los más empleados: random amplified polymorphic DNA (RAPD), amplified fragment length polymorphism (AFLP), simple sequence repeats (SSR) y Ribosomal DNA e internal transcribed spacers (rDNA-ITS), entre otros (Gupta et al., 1999). Con base a lo anterior se realizó este estudio con el objetivo de evaluar la diversidad genética, presente en el ADN de aislados de $P$. capsici, obtenidos en las principales áreas productoras de chile en Guanajuato.

\section{Cepas}

Se utilizaron 30 aislados de $P$. capsici obtenidos de plantas con síntomas de 'marchitez', en 12 lotes de producción comercial de diferentes variedades e híbridos de cincos tipos de chile (poblano, güero, serrano, jalapeño y chilaca), ubicados en los municipios más importantes en la producción de este cultivo en Guanajuato: Dolores Hidalgo, San Luis de la Paz, Juventino Rosas, Silao, Salvatierra y Cortázar (Cuadro 1). Estos aislados fueron identificados usando las claves de Erwin y Ribeiro (1996). Además, de dos cepas PCT17 y PCC6, donadas por la Dra. Sylvia Patricia Fernández Pavía del Laboratorio de Patología Vegetal-UMSNH. 
Cuadro 1. Municipio de origen y tipo de chile donde fueron obtenidos 30 cultivos monozoospóricos de Phytophthora capsici.

\begin{tabular}{cccccc}
\hline Municipio & Clave & Tipo de chile & Municipio & Clave & Tipo de chile \\
\hline Dolores Hidalgo & D1 & Poblano & Silao & S1 & Güero \\
& D2 & Poblano & & S2 & Güero \\
& D3 & Poblano & & S3 & Güero \\
& D4 & Poblano & & S4 & Serrano \\
& D5 & Poblano & & S5 & Jalapeño \\
& D6 & Poblano & & S6 & Jalapeño \\
& D7 & Poblano & & S7 & Jalapeño \\
& D8 & Poblano & Juventino Rosas & J1 & Jalapeño \\
& D9 & Poblano & & J2 & Poblano \\
& D10 & Poblano & & J3 & Jalapeño \\
& D11 & Poblano & Cortázar & CT1 & Jalapeño \\
& D12 & Poblano & & CT3 & Jalapeño \\
& SP1 & Poblano & & CT4 & Jalapeño \\
& SP2 & Poblano & Salvatierra & SA2 & Chilaca \\
& SP3 & Poblano & & SA3 & Chilaca \\
\hline
\end{tabular}

De todos los aislados incluyendo las cepas de referencia se hicieron cultivos monozoospóricos de acuerdo con el protocolo de Mitchell y Kannwischer-Mitchell (1995); Erwin y Ribeiro (1996). Los cultivos se mantuvieron en medio sólido V8C (160 ml de jugo V8, 3 g CaCO3 y $840 \mathrm{ml}$ de agua desionizada), transfiriéndolos cada seis semanas.

\section{Diversidad genética}

El ADN de los 30 aislados y las dos cepas de referencia, se obtuvo a partir de micelio de los cultivos monozoospóricos, utilizando el método propuesto por Doyle y Doyle (1987). Se cuantificó con la ayuda de un espectrofotómetro (NanoDrop 8000) y se uniformizaron las diluciones a una concentración de $120 \mathrm{ng}^{\mu \mathrm{l}^{-1}}$ de $\mathrm{ADN}$.

Los polimorfismos en la longitud de los fragmentos amplificados (AFLP), se detectaron con base en el protocolo descrito por Vos et al. (1995), utilizando un secuenciador NEN Global IR2, marca LI-COR, que genera y permite la captura automática de la imagen digital. Los iniciadores adaptador-sitio de restricción Eco R1 (E+2) usados en este trabajo fueron: E+AT, E+AG y E+AC; los iniciadores adaptador-sitio de restricción Tru $91(\mathrm{~T}+2)$ fueron $\mathrm{T}+\mathrm{CC}, \mathrm{T}+\mathrm{CA}$ y $\mathrm{T}+\mathrm{CG}$. Con estos iniciadores se formaron las seis combinaciones usadas para el análisis: E+AT/T+CC, E+AT/T+CA, $\mathrm{E}+\mathrm{AG} / / \mathrm{T}+\mathrm{CC}, \mathrm{E}+\mathrm{AG} / \mathrm{T}+\mathrm{CA}, \mathrm{E}+\mathrm{AG} / \mathrm{T}+\mathrm{CG}, \mathrm{y} \mathrm{E}+\mathrm{AC} / \mathrm{T}+\mathrm{CG}$.

Las imágenes generadas, se analizaron usando el programa 'Cross Checker 2.91' que codifica las bandas usando un código binario donde cada banda se representa como presente (1) o ausente (0), se consideró que un fragmento de $\mathrm{ADN}$ es monomórfico, si $99 \%$ de la población lo tiene y polimórficos a aquellos que difieren de este porcentaje (Cavalli-Sforza and Bodmer; 1981). Los 
datos binarios fueron analizados con el programa NTSYS-pc versión 2.1 (Numerical Taxonomy Multivariate Analysis System) (Rohlf, 2005) para generar una matriz de similitud basada en el coeficiente de Dice (Núñez-Colín y Valadez-Moctezuma, 2010), el cual considera para determinar la semejanza entre dos aislados solo las coincidencias. Se realizó un procedimiento Bootstrap con 2000 remuestreos con remplazo, a partir de la matriz de similitud promedio se determinaron las relaciones genéticas entre los genotipos estudiados generando un dendrograma consenso aplicando el método Unweighted Pair Group Method using Arithmetic Average (UPGMA) (Sneath and Sokal, 1973).

Se consideraron para el análisis solo las bandas bien definidas y de mediana a alta intensidad (Figura 1). Se obtuvieron en total 807 bandas de las cuales $78.81 \%$ fueron polimórfica. El porcentaje de polimorfismo de las bandas es similar al reportado por Kersey et al. (2005) quienes al analizar 31 aislados de $P$. capsici de Nuevo México EU., encontraron que 80\% de 1155 bandas fueron polimórficas. El nivel de polimorfismo fue mayor que el obtenido por Lamour y Hausbeck (2001a) para aislados de P. capsici de Michigan EU., quienes reportaron un porcentaje promedio de polimorfismos de $43 \%$ para 94 bandas.

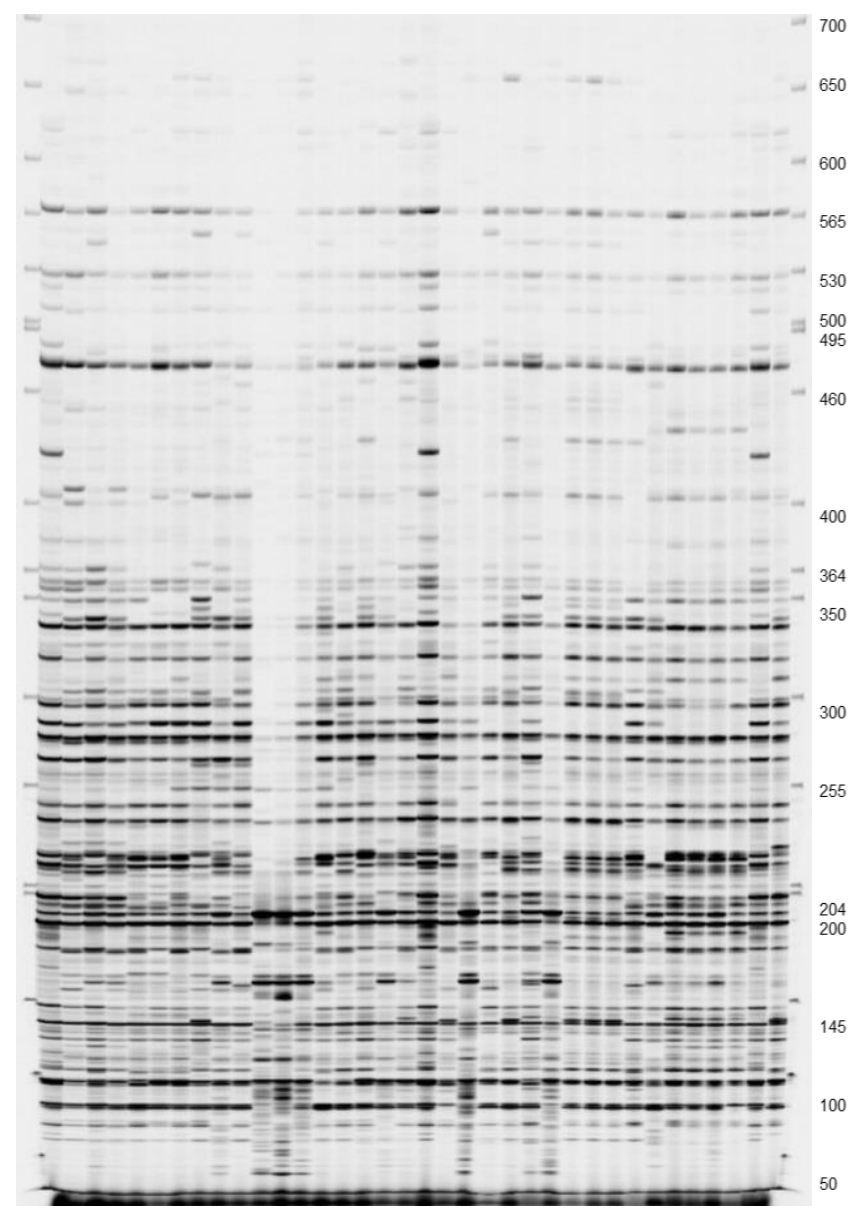

Figura 1. Polimorfismos en la longitud de los fragmentos amplificados (AFLP), por sus siglas en inglés, perfiles genéticos generados por la combinación de iniciadores $\mathrm{E}+\mathrm{AT} / \mathrm{T}+\mathrm{CA}$ en 30 aislados de $\boldsymbol{P}$. capsici del estado de Guanajuato y dos cepas de referencia, que muestra la definición y la intensidad de los fragmentos de ADN (bandas) amplificados. 
La matriz de similitud basada en el coeficiente de Dice, muestra valores que van de 76 a $95 \%$ de similitud con un promedio de $86 \%$, estas relaciones genéticas se ven reflejadas en el dendrograma consenso obtenido en este trabajo (Figura 2). En él se puede apreciar la formación de cuatro grupos bien definidos, el grupo I formado con dos aislados de Cortázar y uno de Silao los cuales presentan la menor similitud con el resto; los agrupamientos con la mayor cantidad de aislados son el grupo II formado solo por aislados de Dolores Hidalgo y el grupo III formado por una mezcla de aislados de Silao y Dolores Hidalgo, el grupo IV está formado por seis subgrupos, resaltando que cinco de ellos están formados solo con aislados del mismo municipio, estos son los subgrupos formados solo con aislados de Silao, Dolores Hidalgo, San Luis de la Paz, Juventino Rosas y el subgrupo formado por las dos cepas de referencias las cuales siguiendo la tendencia observada, podrían provenir de una misma localidad.

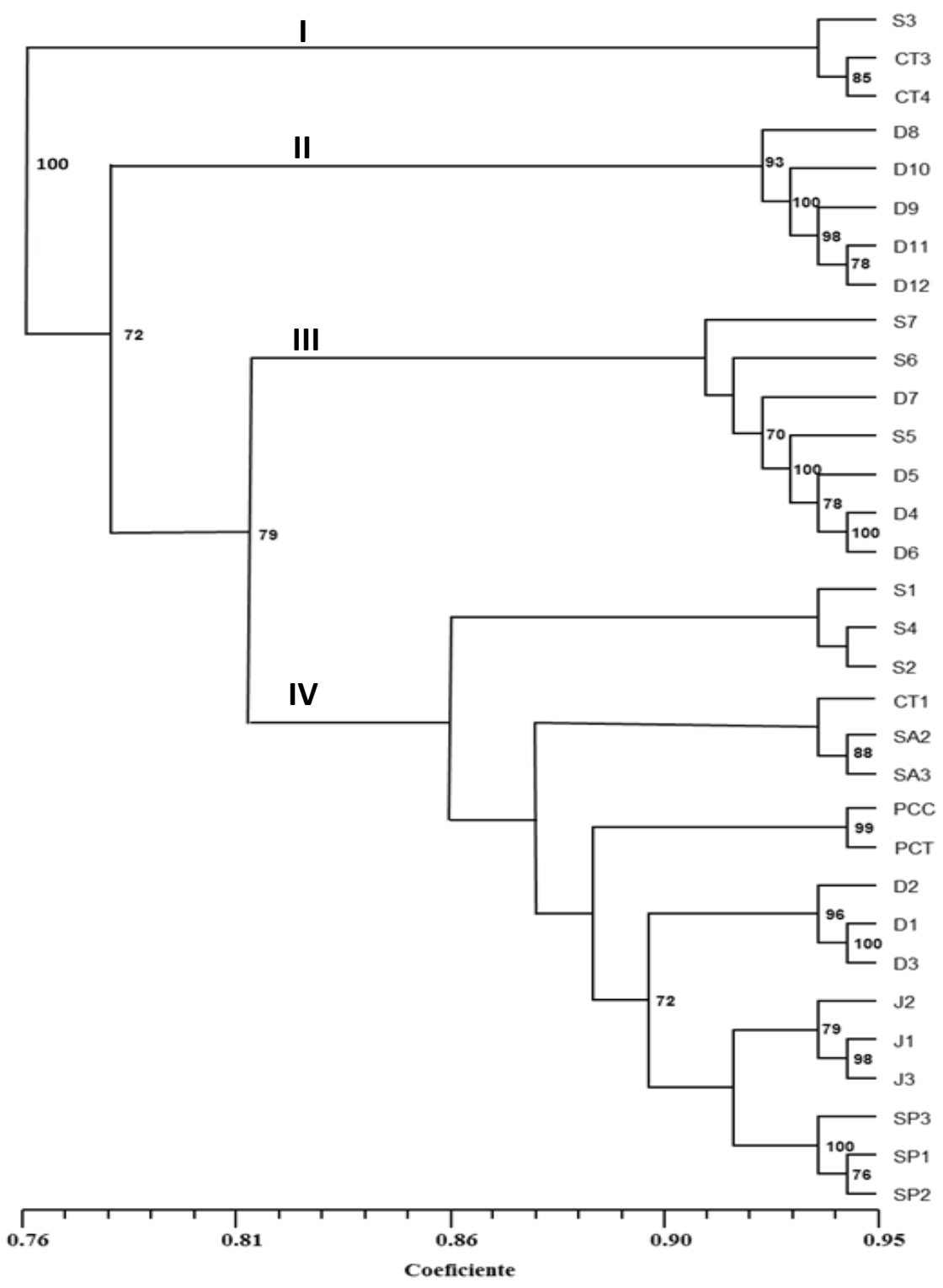

Figura 2. Dendrograma consenso de 30 aislados de $P$. capsici del estado de Guanajuato y dos cepas de referencia. Los números en los nodos son el porcentaje de dendrogramas por encima de $70 \%$ que fueron respaldados por el análisis bootstrap. 
En este grupo solo se formó un subgrupo que mezcla dos cepas originarias de Salvatierra con una de Cortázar. Estos resultados parecen indicar una tendencia de los aislados a agruparse de acuerdo con su municipio de origen. Excepto los cultivos monozoospóricos originarios de Silao y Cortázar que se agruparon con aislados de diferentes localidades, lo cual es indicativo de una alta diversidad genética.

La asociación basada en el lugar de aislamiento concuerda con el estudio de variabilidad de aislados de P. capsici originarios de cuatro diferentes regiones de Michigan EU. reportado por Lamour y Hausbeck (2001a), en el cual el agrupamiento encontrado se basó en el sitio de colecta. Igualmente, en lo reportado por Parada-Rojas y Quesada-Ocampo (2018) quienes usando microsatélites para analizar las relaciones genéticas de 50 aislados de $P$. capsici originarios de varios estados de EU., encuentran una estructura de la población basada en el origen geográfico. En México, CastroRocha et al. (2016) analizando la diversidad de 80 aislados originarios del norte y centro de este país, usando marcadores tipo SNP, señalan que los aislados de Chihuahua forman dos grupos y el resto forma tres grupos estrechamente relacionados, compuestos principalmente de aislamientos de Aguascalientes, Guanajuato y Michoacán, encontrando una separación por regiones de aislamiento.

Si se considera lo señalado por Brasier y Hansen (1992), de que el género Phytophthora, tiene una fuerte selección por el clima, por lo que la mayoría de sus especies, se encuentran en zonas de temperaturas frescas subtropicales o tropicales y que la humedad, el contenido de nutrientes, el $\mathrm{pH}$ y microorganismos nativos, son factores que influyen en la adaptación de este patógeno. Entonces, pareciera ser que las diferencias entre las condiciones ambientales de las regiones de Guanajuato en donde se realizaron las colectas son los principales factores que influyeron en la formación de los grupos en este estudio.

Los municipios del norte del estado, San Luis de la Paz y Dolores Hidalgo, cuentan con un clima semiseco, con una precipitación anual de $387.5 \mathrm{~mm}$ y temperatura media anual de $16{ }^{\circ} \mathrm{C}$; Silao, Juventino Rosas y Cortázar que se encuentran en centro del estado, tienen un clima subtropicalsubhúmedo con una precipitación anual de $688 \mathrm{~mm}$ y temperatura media anual de $19.4{ }^{\circ} \mathrm{C}$, muy parecido a las condiciones climáticas de Salvatierra que está al sur del estado, con clima predominante templado húmedo con una precipitación anual de $730 \mathrm{~mm}$ y temperatura media anual de $18.1^{\circ} \mathrm{C}$ (INEGI, 2013). Las inconsistencias del agrupamiento por lugar de aislamiento podrían ser explicadas por efectos de muestreo, deriva genética o por entrecruzamiento en poblaciones pequeñas, lo que puede provocar cambios en la diversidad genética (Goodwin, 1997) y por consiguiente cambios en los agrupamientos.

Todos los aislados de $P$. capsici de Guanajuato, fueron genotipos únicos, esto era lo esperado por los resultados obtenidos en el proyecto de secuenciación del genoma, en donde reportan que la densidad y diversidad de las variantes de un solo nucleótido entre genomas de $P$. capsici es notablemente más alta que en otros genomas eucariotas, lo que ha confirmado que los aislados de $P$. capsici, tienen una gran variación genética en la forma de SNPs, los cuales pueden ocurrir tan frecuentemente como 1 cada 40 pb (Lamour et al., 2012).

Al incluir en el análisis sus características morfológicas y fisiológicas descritas en un trabajo previo (Pons et al., 2020), no se encontró una asociación clara entre los grupos genéticos con la morfología, tipo de compatibilidad, sensibilidad a mefenoxam, el tipo de chile o el grado de 
virulencia. Esta ausencia de correspondencia fue encontrada en otros Phytophthora spp. (Lebreton y Andrivon, 1998; Mahuku et al., 2000; Abu-El Samen et al., 2003) en donde no se encontró ninguna correlación entre los grupos definidos por marcadores moleculares y la virulencia, tipo de apareamiento o respuesta a metalaxil de los aislados analizados.

A pesar de la ausencia de estas correlaciones, los resultados de este trabajo nos señalan que la población de Phytophthora capsici de Guanajuato mantienen su característica de altos niveles de diversidad, como lo han reportado en otras poblaciones de este patógeno varios autores (Lamour y Hausbeck 2001a; Hausbeck y Lamour 2004; Gevens et al., 2008; Hurtado-Gonzáles et al., 2008; Meitz et al., 2010; Lamour et al., 2011; Gobena et al., 2012). Como se encontraron los dos tipos de apareamiento en varios de los municipios de colecta (Pons et al., 2020), es probable que una parte de esta diversidad resulte de la recombinación sexual y otra de mutaciones que, de acuerdo con lo reportado por Goodwin (1997), es la fuente primaria de la nueva diversidad genética en oomicetos. Independientemente de su origen, la presencia de diversidad genética en la población de $P$. capsici de Guanajuato, indica que existe un potencial genético en la población de patógenos para el desarrollo de resistencia a fungicidas y sobrepasar las defensas del hospedero, por lo que es necesario monitorearla continuamente y considerarla para el diseño de estrategias para su control.

\section{Conclusiones}

Existe variabilidad genotípica entre los aislados de P. capsici de Guanajuato. Todos los aislados de $P$. capsici de Guanajuato, son genotipos únicos ya que no se encontraron individuos clonales. Los grupos genéticos que se forman están basados en el lugar de origen de los aislados. No se encontró una asociación clara entre los grupos genéticos y la virulencia, tipo de apareamiento o respuesta a metalaxil en los aislados analizados.

\section{Agradecimientos}

Los resultados son parte del proyecto fiscal: Desarrollo y transferencia de tecnologías sustentables para la producción de chile y jitomate en campo e invernadero, núm. 167834791.

\section{Literatura citada}

Abu-El Samen, F. M.; Secor, G. A. and Gudmestad, N. C. 2003. Genetic variation among asexual progeny of Phytophthora infestans detected with RAPD and AFLP markers. Plant Pathol. 52(3):314-325. https://doi.org/10.1046/j.1365-3059.2003.00858.x.

Brasier, C. M. and Hansen, E. 1992. Evolutionary biology of Phytophthora Part II: phylogeny, speciation, and population structure. Annual Rev. Phytopathol. 30(1):173-200. https://doi.org/10.1146/annurev.py.30.090192.001133.

Castro-Rocha, A.; Shrestha, S.; Lyon, B.; Grimaldo-Pantoja, G. L.; Flores-Marges, J. P.; ValeroGalván, J.; Aguirre-Ramírez, M.; Osuna-Ávila, P.; Gómez-Dorantes, N.; Ávila-Quezada, G.; Luna-Ruíz, J. J.; Rodríguez-Alvarado, G.; Fernández-Pavía, S. P. and Lamour, K. 2016. An initial assessment of genetic diversity for Phytophthora capsici in northern and central Mexico. Mycol Progress. 15(2):1-12. Doi 10.1007/s11557-016-1157-0.

Cavalli-Sforza, L. L. y Bodmer, W. F. 1981. Genética de las poblaciones humanas. (Ed.). Omega, Barcelona. 124-126 pp. 
Doyle, J. J. and Doyle, J. L. 1987. A rapid DNA isolation procedure from small quantities of fresh leaf tissues. Phytochem. Bulletin. 19(1):11-15. http://irc.igd.cornell.edu/protocols/Doyle Protocol.pdf.

Erwin, D. C. and Ribeiro, O. K. 1996. Phytophthora Diseases Worldwide. APS PRESS. The American Phytopathological Society. St. Paul, Minnesota. 562 p.

Gevens, A. J.; Donahoo, R. S.; Lamour, K. H. and Hausbeck, M. K. 2008. Characterization of Phytophthora capsici causing foliar and pod blight of snap bean in Michigan. Plant Dis. 92(2):201-209. Doi:10.1094/PDIS-92-2-0201.

Gobena, D.; Roig, J.; Galmarini, C.; Hulvey, J. and Lamour, K. H. 2012. Genetic diversity of Phytophthora capsici isolates from pepper and pumpkin in Argentina. Mycologia. 104(1):102-107. Doi:10.3852/11-147.

Goodwin, S. B. 1997. The population genetics of Phytophthora. Phytopathology. 87(4):462-473. Doi: 10.1094/PHYTO.1997.87.4.462.

González-Pérez, E.; Yáñez-Morales, M.; Santiago-Santiago, V. y Montero-Pineda, A. 2004. Biodiversidad fungosa en la marchitez del chile y algunos factores involucrados, en Tlacotepec de José Manzo, El Verde, Puebla. Agrociencia. 38(6):653-661. http://www.redalyc.org/articulo.oa?id=30238609.

Granke, L. L.; Windstam, S. T.; Hoch, H. C.; Smart, C. D. and Hausbeck, M. K. 2009. Dispersal and movement mechanisms of Phytophthora capsici sporangia. Phytopathology. 99(11):1258-1264. https://doi.org/10.1094/Phyto-99-11-1258.

Granke, L. L.; Quesada-Ocampo, L. and Lamour, K. 2012. Advances in research on Phytophthora capsici on vegetable crops in the United States. Plant Dis. 95(11):1588-1600. https://doi.org/10.1094/PDIS-03-11-0190.

Gupta, P. K.; Varshney, R. K.; Sharma, P. C. and Ramesh, B. 1999. Molecular markers and their applications in wheat breeding. Plant Breed. 118(5):369-390. https://doi.org/10.1046/ j.1439-0523.1999.00401.x.

Hausbeck, M. K. and Lamour, K. H. 2004. Phytophthora capsici on vegetable crops: research progress and management challenges. Plant Dis. 88(12):1292-1303. https://doi.org/ 10.1094 /PDIS.2004.88.12.1292.

Hurtado-Gonzáles, O.; Aragón-Caballero, L.; Apaza-Tapia, W.; Donahoo, R. and Lamour, K. 2008. Survival and spread of Phytophthora capsici in Coastal Peru. Phytopathology. 98(6):688-694. https://doi.org/10.1094/Phyto-98-6-0688.

INEGI. 2013. Instituto Nacional de Estadística y Geografía. Conociendo Guanajuato. Aguascalientes, Aguascalientes. México. Serie Conociendo México. Folleto informativo. $30 \mathrm{p}$.

Kersey, R.; Sanogo, S.; Carpenter, J. and Bosland, P. 2005. Determination of genetic diversity of Phytophthora capsici isolates in New Mexico with AFLP Markers. in Plant \& Animal Genomes XIII Conference. 15-19 pp.

Lamour, K. H. and Hausbeck, M. K. 2001a. The dynamics of mefenoxam insensitivity in a recombining population of Phytophthora capsici characterized with amplified fragment length polymorphism markers. Phytopathology. 91(6):553-557. https://doi.org/10.1094/ Phyto.2001.91.6.553.

Lamour, K. H. and Hausbeck, M. K. 2001b. Investigating the spatiotemporal genetic structure of Phytophthora capsici in Michigan. Phytopathology. 91(10):973-980. https://doi.org/10. 1094/PHYTO.2001.91.10.973. 
Lamour, K. H.; Stam, R.; Jupe, J. and Huitema, E. 2011. The oomycete broad-host-range pathogen Phytophthora capsici. Mol. Plant Pathol. 13(4):329-337. Doi: 10.1111/j.13643703.2011.00754.x.

Lamour, K.; Mudge, J.; Gobena, D.; Hurtado-Gonzáles, O.; Shmutz, J.; Kuo, A.; Miller, N. A.; Rice, B. J.; Raffaele, S.; Cano, L. M.; Bharti, A. K.; Donahoo, R. S.; Finley, S.; Huitema, E.; Hulvey, J.; Platt, D.; Salamov, A.; Savidor, A.; Sharma, R.; Stam, R.; Storey, D.; Thines, M.; Win, J.; Haas, B. J.; Dinwiddie, D. L.; Jenkins, J.; Knight, J. R.; Affourtit, J. P.; Han, C. S.; Chertkov, O.; Lindquist, E. A.; Detter, C.; Grigoriev, I. V.; Kamoun, S. and Kingsmore, S. K. 2012. Genome sequencing and mapping reveal loss of heterozygosity as a mechanism for rapid adaptation in the vegetable pathogen Phytophthora capsici. Mol. Plant-Microbe Interact. 25(10):1350-1360. Doi:10.1094/MPMI-02-12-0028-R.

Lebreton, L. and Andrivon, D. 1998. French isolates of Phytophthora infestans from potato and tomato differ in phenotype and genotype. Eur. J. Plant Pathol. 104(6):583-594. Doi:10.1023/A:1008662518345.

Martin, F. N.; Abad, Z. G.; Balci, Y. and Ivors, K. 2012. Identification and Detection of Phytophthora: Reviewing Our Progress, Identifying Our Needs. Plant Dis. 96(8):10801103. https://doi.org/10.1094/PDIS-12-11-1036-FE.

Mahuku, G.; Peters, R. D.; Platt, H. W. and Daayf, F. 2000. Random amplified polymorphic DNA (RAPD) analysis of Phytophthora infectants isolates collected in Canada during 1994 to 1996. Plant Pathol. 49(2):252-260. https://doi.org/10.1046/j.1365-3059.2000.00450.x.

McDonald, B. A. and Linde, C. 2002. Pathogen population genetics, evolutionary potential, and durable resistance. Annu. Rev. Phytopathol. 40(1):349-379. https://doi.org/10.1146/ annurev.phyto.40.120501.101443.

Meitz, J. C.; Linde, C. C.; Thompson, A.; Langenhoven, S. and McLeod, A. 2010. Phytophthora capsici on vegetable hosts in South Africa: distribution, host range and genetic diversity. Australasia Plant Path. 39(5):431-439. https://doi.org/10.1071/AP09075.

Mitchell, D. J. and Kannwischer-Mitchell, M. E. 1995. Genetic, biochemical, and molecular techniques for the identification and detection of soilborne plant-pathogenic fungi. In: methods for research on soilborne phytopathogenic fungi. (Ed.). Singleton, L. L.; Mihail, J. D. and Crush, M. APS Press., USA. 95-98 pp.

Mohammadi, S. A. and Prasanna, B. M. 2003 Review and interpretation analysis of genetic diversity in crop plants-salient statistical tools. Crop Sci. 43(4):1235-1248. https://doi.org/10.2135/cropsci2003.1235.

Núñez-Colín, C. A. y Valadez-Moctezuma, E. 2010. Análisis estadístico de huellas genómicas. Un uso práctico de los paquetes computacionales más populares. INIFAP, Celaya, Guanajuato, México. 103 p.

Parada-Rojas, C. H. and Quesada-Ocampo, L. M. 2018. Analysis of microsatellites from transcriptome sequences of Phytophthora capsici and applications for population studies. Scientific Reports. 8(5194):1-12. https://doi.org/10.1038/s41598-018-23438-8.

Pons, H. J. L.; Guerrero-Aguilar, B. Z.; González-Chavira, M. M.; González-Pérez, E.; VillalobosReyes, S. y Muñoz-Sánchez, C. I. 2020. Variabilidad fenotípica de aislados de Phytophthora capsici en Guanajuato. Rev. Mex. Cienc. Agríc. 11(8):1891-1901. https://doi.org/10.29312/remexca.v11i8.2618.

Quesada-Ocampo, L. M.; Granke, L. L.; Mercier, M. R.; Olsen, J. and Hausbeck, M. K. 2011. Investigating the genetic structure of Phytophthora capsici populations. Phytopathology. 101(9):1061-1073. Doi: 10.1094/Phyto-11-10-0325. 
Rohlf, F. J. 2005. NTSYSpc: numeral taxonomy and multivariate analysis system. Ver. 2.2. Exeter Software. Setauket, USA. 42 p.

Sneath, P. H. A. and Sokal, R. R. 1973. Numerical taxonomy-the principle and practice of numerical classification. Freeman, W. H. and Co., San Francisco. https://doi.org/10.2307 12412767.

Universidad Illinois. 2001. Phytophthora blight of pepper. Report on plant disease. Department of crop sciences, University of Illinois at Urbana-Champaign. RPD. 947 p. http://web.aces.uiuc.edu/vista/pdf_pubs/947.pdf.

Vos, P.; Hogers, R.; Bleeker, M.; Reijans, M.; Van der Lee, T.; Hornes, M.; Frijters, A.; Pot, J.; Peleman, J.; Kulper, M. and Zabeau, M. 1995. AFLP: a new technique for DNA fingerprinting. Nucleic Acids Res. 23(1):4407-4414. Doi: 10.1093/nar/23.21.4407. 